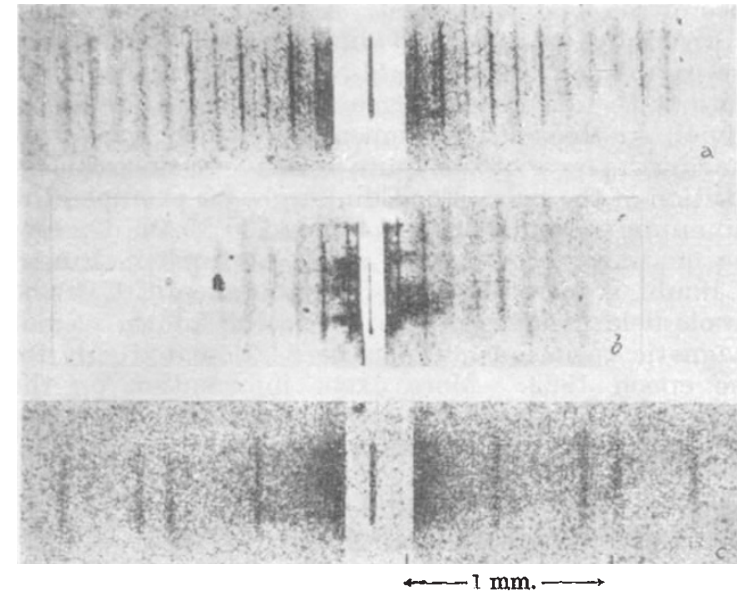

Enlargements of low-angle X-ray diffraction photographs. (a) Collagen from kangaroo tail tendon, $3 \mathrm{hr}$. exposure at $50 \mathrm{kV}$; $450 \mu \mathrm{mp}$. The repeat distance is $640 \mathrm{~A}$.; the second order $(17$ deflexion) is clearly visible. (b) Collagen from stained rat tail 30 min. exposure at $50 \mathrm{k} ., 450 \mu \mathrm{A}$. The hrst order ( 8 (c) Texion) is visible mosalc virus gel, $130 \mathrm{~A}$.

few orders appearing within $30 \mathrm{~min}$. With favourable specimens such as collagen stained with phosphotungstic acid, the range of visibility goes up to twice this figure, because the exposure times are short, the first few orders appearing within $5 \mathrm{~min}$. Fig. $b$ is not fully representative, as it has been obtained with the width of the slit near the specimen below its optimum value. This increases the first-order visibility somewhat and the exposure-time by about a factor 3 .

W. EHRENBERG

A. Franks

Birkbeck College

(University of London),

Malet Street,

London, W.C.1.

Aug. 28.

${ }^{1}$ Bear, J., J. Amer. Chem. Soc., 66, 1297 (1944).

? Guinier, A., and Fournet, G., C.R. Acad. Sci., Paris, 226, 656 (1948)

S "The Development of Focusing X-ray Cameras for Use at Small Diffraction Angles" (Mass. Inst. Tech. thesis, 1952).
Us, Ther Ehrenberg, W., J. Opt. Soc. Amer., 39, 741 (1949).

${ }^{5}$ Ehrenberg, W., and Spear, W. E., Proc. Phys. Soc., B, 64, 67 (1951).

\section{Behaviour of Atmospheric Pressure Systems}

A METHOD for studying the behaviour of atmospheric pressure systems has lately been developed at the local weather bureau at Pretoria. The general method, which can be applied in a number of different ways, can best be explained by a concrete example.

The departures (at a fixed hour) of daily pressure from the appropriate means were tabulated for 10-deg. grid points and expressed as percentages of the respective standard deviations. A grid point $P$, at $80^{\circ} \mathrm{W}$., $50^{\circ} \mathrm{N}$., was selected, and whenever the departure exceeded the standard deviation and formed a point of inflexion, the departure being greater than on the day before and on the day after, the date was written down. This occurred on 380 days during a period of ten years, 1929-38. The mean pressure for these 380 days was computed at $P$ and at a large number of neighbouring grid points. The resulting mean isobars reveal an extremely simple pattern, since at all points sufficiently remote from $P$, the mean departure of pressure is practically zero.

The behaviour of this simplified isobaric pattern with the passage of time can now be readily studied. If one day be added to all the listed dates and the average pressure found for the new set of 380 dates, we obtain a chart of the mean isobars $24 \mathrm{hr}$. later.

In order further to simplify the picture of what actually happens, the pressures are shown graphically only for the 50th parallel north. Curve $A$ was drawn to fit the original pressures as computed and shown in the accompanying figure. Curves $B$ and $C$ represent the distribution after one and two days respectively, the units of pressure used being quite arbitrary.

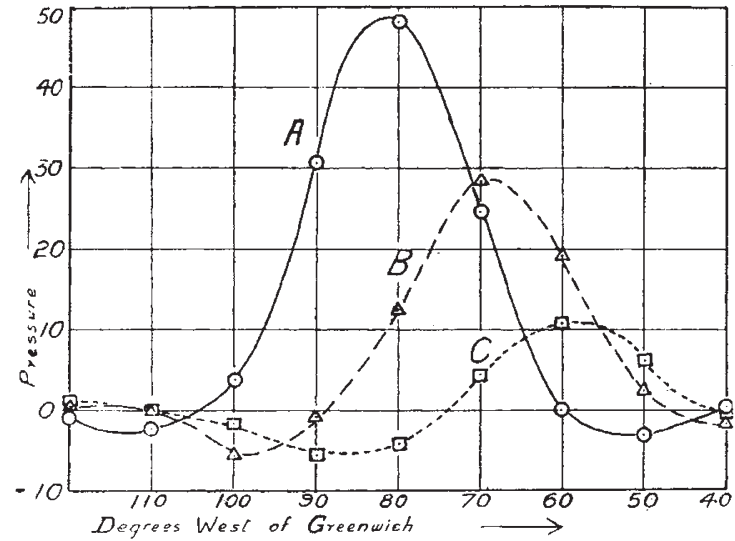

As one would expect, the isobaric pattern moves from west to east due to the prevailing westerlies. In this case careful calculation indicates a displacement of $10.7 \mathrm{deg}$. or 413 nautical miles in $24 \mathrm{hr}$., equivalent to a speed of $17.2 \mathrm{knots}$, in close agreement with the known rate of travel of pressure systems.

The curves presented here show several other features, only one of which need be discussed at the moment. When the temperature distribution curves in a conducting rod, resulting from an instantaneous point source of heat, are graphically represented after fixed intervals of time, they show a striking similarity with curves $A, B$ and $C$, except that the latter are displaced eastwards with the passage of time. It would therefore appear to be quite legitimate to introduce the concept of the 'diffusion of atmospheric pressure', and it should not be impossible to derive the laws of pressure diffusion even though they are bound to be appreciably more complicated than the analogous law of diffusion of heat. If these laws are reasonably well known, they could be applied to determine to a first approximation to-morrow's pressure at any point on the basis of to-day's pressure distribution. The preparation of prebaratic charts, as currently drawn at most forecasting centres, should thus be facilitated.

Research on the lines indicated is being continued at Pretoria and the results will be published in due course.

\section{T. SchumanN}

(Director)

Department of Transport, Weather Bureau, Pretoria. June 5 . 